
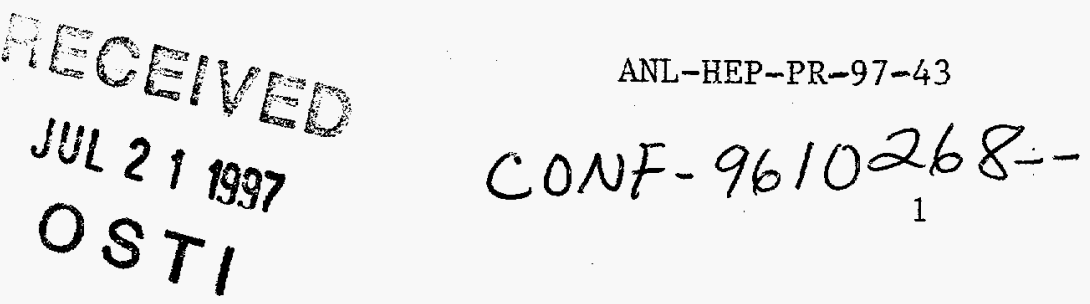

\title{
Detectors for the MINOS long-baseline neutrino oscillation experiment *
}

\author{
David S. Ayres ${ }^{\mathrm{a}}$ \\ ${ }^{2}$ High Energy Physics Division, Argonne National Laboratory, \\ Argonne, Illinois 60439 , U.S.A.
}

The MINOS long-baseline experiment will use an intense neutrino beam, generated by Fermilab's Main Injector accelerator, and a $730 \mathrm{~km}$ flight path to search for neutrino oscillations. The 10,000 ton MINOS far detector will utilize toroidally magnetized steel plates interleaved with track chambers to reconstruct event topologies and to measure the energies of the muons, hadrons and electromagnetic showers produced by neutrino interactions. The MINOS collaboration is currently developing three alternative technologies for the track chambers: 'Iarocci' tubes (operated in either limited streamer or saturated proportional mode), RPC's (with either glass or ABS plates), and scintillator (either liquid or plastic) with wavelength shifting fiber readout. The technology choice will be made in mid 1997 based on the projected performance and cost of the $32,000 \mathrm{~m}^{2}$ active detector system.

\section{Introduction}

The MINOS (Main Injector Neutrino Oscillation Search) experiment will search for the oscillations of muon neutrinos into tau and/or electron neutrinos in an intense neutrino beam generated by Fermilab's new Main Injector accelerator. The experiment [1] is being mounted by the MINOS collaboration [2], which currently consists of 200 physicists and engineers from 22 institutions in the United States, the United Kingdom, Russia, and China. The $10 \mathrm{GeV}$ neutrino beam energy is well above threshold for tau leptons, which would be produced if $\nu_{\mu} \rightarrow \nu_{\tau}$ oscillations should occur. The design of the neutrino beam will allow operation in both wide-band and narrow-band configurations, in order to verify and explore any oscillation signal which is observed.

Oscillations will be detected by comparison of neutrino event characteristics (e.g., rate, energy spectrum, NC/CC ratio) in a 'near' detector at Fermilab and a 10,000 ton 'far' detector situated $730 \mathrm{~km}$ away in the Soudan underground laboratory [3]. The sensitivity of the oscillation search will depend on the ability to predict the characteristics of the neutrino beam at the far detector in the absence of oscillations. Systematic errors

\footnotetext{
*Submitted to the Proceedings of the 5th International Conference on Advanced Technology and Particle Physics, Como, Italy, 7-11 October 1996.
}

from detector response effects will be minimized by making the near and far detectors as similar as possible. Measurements of neutrino events in the 1000-ton, very fine grained Soudan 2 tracking calorimeter, which is currently taking data on atmospheric neutrino interactions and searching for nucleon decay, will complement those in the much larger, coarser grained MINOS detector at Soudan.

The initial feasibility studies for the experiment made use of a 'reference' detector design whose performance and construction cost could be predicted with some confidence. The 10,000 ton far reference detector consists of 6004 -cm thick magnetized iron planes interleaved with $32,000 \mathrm{~m}^{2}$ of limited streamer Iarocci tubes, read out by 480,000 electronics channels. The 8-m wide octagonal steel planes are toroidally magnetized [4] with an average field of $1.5 \mathrm{~T}$ by a low power conventional magnet coil which passes through a central hole. The 1-cm square Iarocci tubes are read out by their anode wires (summed in pairs) and $2-\mathrm{cm}$ wide external cathode strips. The estimated cost of the near and far reference detectors is $\$ 50 \mathrm{M}$. The far reference detector is shown schematically in Fig. 1.

Three different active detector technologies are now being considered for the MINOS far detector. Since the approval of the experiment in September 1995, the design of the reference de- 


\section{DISCLAIMER}

This report was prepared as an account of work sponsored by an agency of the United States Government. Neither the United States Government nor any agency thereof, nor any of their employees, make any warranty, express or implied, or assumes any legal liability or responsibility for the accuracy, completeness, or usefulness of any information, apparatus, product, or process disclosed, or represents that its use would not infringe privately owned rights. Reference herein to any specific commercial product, process, or service by trade name, trademark, manufacturer, or otherwise does not necessarily constitute or imply its endorsement, recommendation, or favoring by the United States Government or any agency thereof. The views and opinions of authors expressed herein do not necessarily state or reflect those of the United States Government or any agency thereof. 


\section{DISCLAIMER}

Portions of this document may be illegible electronic image products. Images are produced from the best available original document. 


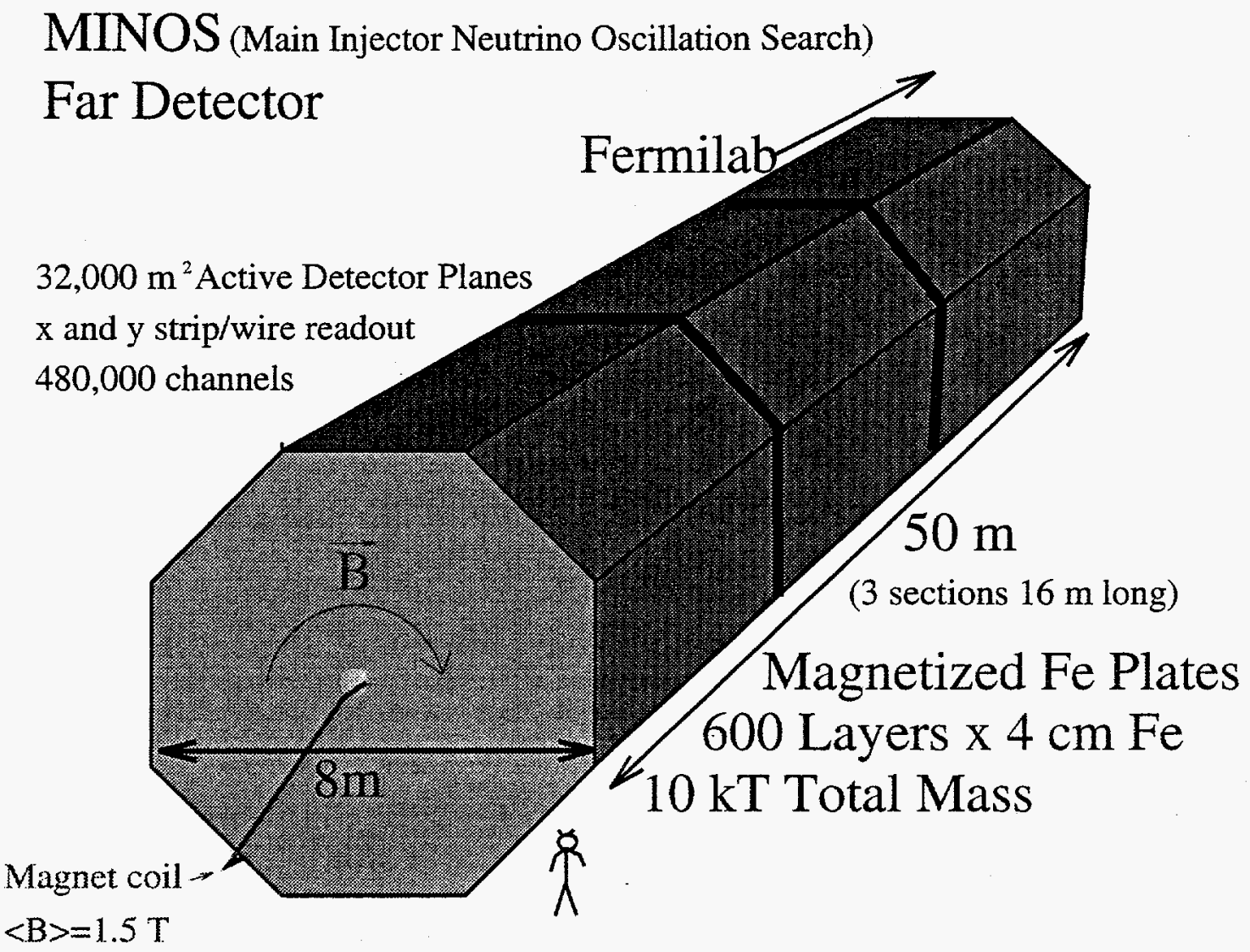

Figure 1. The MINOS reference detector, located $700 \mathrm{~m}$ underground in Soudan, Minnesota.

tector Iarocci tubes has been optimized for the experiment. In addition, two alternative technologies are being developed: resistive plate counters (RPC's), and scintillator (either plastic or liquid) read out with wavelength shifting fibers. One of these technologies will be chosen in mid 1997 based on projected performance and cost. The detector design will be frozen in the fall of 1997 so that the first third of the far detector can be ready for beam turnon in the summer of 2001 .

\section{Detector requirements}

The MINOS detector design is optimized for the study of $\nu_{\mu} \rightarrow \nu_{\tau}$ oscillations with $\sin ^{2}(2 \theta) \geq$ 0.025 and $\Delta m^{2} \geq 0.001 \mathrm{eV}^{2}$. The $\sin ^{2}(2 \theta)$ sen- sitivity improves to 0.01 for $\nu_{\mu} \rightarrow \nu_{e}$ oscillations. If oscillations are observed, the experiment will determine the oscillation mode, or mixture of modes, and will measure the $\sin ^{2}(2 \theta)$ and $\Delta m^{2}$ parameters for each mode. The identification of tau leptons produced in $\nu_{\tau}$ charged current interactions is difficult because 'tails' of the beam energy spectrum and detector resolution functions can cause serious backgrounds. Currently several techniques for identifying tau lepton decays in different modes are under study, using accurate simulations of the neutrino beam and detector response functions.

The ability to identify CC (charged current) interactions caused by electron or tau neutrinos, in 
the presence of a large background of muon neutrino CC and NC (neutral current) events, will be a very important tool in the search for neutrino oscillations. MINOS can easily distinguish between $\nu_{\mu} \mathrm{CC}$ interactions and ' $\mathrm{NC}$ ' events on the basis of a simple event length cut. The 'NC' sample of short events contains most $\nu_{e}$ and $\nu_{\tau}$ $\mathrm{CC}$ events as well as true $\mathrm{NC}$ events. Good spatial resolution and event topology reconstruction will improve the ability to select a subset of the ' $\mathrm{NC}$ ' events which have imbedded EM showers. This subset is enriched in $\nu_{e}$ and $\nu_{\tau} \mathrm{CC}$ events, which will be identified statistically on the basis of simulations and the comparison of far detector event topologies with those in the near detector (where the beam is nearly pure $\nu_{\mu}$ ). In addition, the $\mathrm{CC}$ event sample will contain any $\tau \rightarrow e \nu \nu$ and $\tau \rightarrow \pi \nu$ decay events. For data taken with the narrow-band beam these may be identified on a statistical basis using kinematic constraints.

Any oscillation signal observed can be verified and studied using two or more independent oscillation tests. These involve comparisons between the near and far detectors of quantities such as the neutrino event rate, the $\mathrm{NC} / \mathrm{CC}$ ratio, the energy spectra of $\mathrm{CC}$ and $\mathrm{NC}$ events, electron and (possibly) tau lepton appearance. Each of these tests places different requirements on detector performance, making the design optimization somewhat subjective. The measurement of event energy will be central to the study of any oscillation signal observed; the following resolutions for muons, hadrons, and electromagnetic showers are expected:

$(\Delta p / p)_{\mu} \approx 10 \%$ (range and/or curvature), $(\Delta E / E)_{\text {hadron }} \leq 100 \% / \sqrt{E}$, and $(\Delta E / E)_{e m} \leq 40 \% / \sqrt{E}$.

Since the total detector construction cost is constrained to be $\$ 50 \mathrm{M}$ or less, the detector optimization may involve 'trading off' mass (event rate) against performance, e.g., transverse and longitudinal (steel plane thickness) granularities. The technology for the construction and installation of the steel planes is now reasonably well understood and the first full size prototype plane will be assembled (from two layers of $2 \mathrm{~m}$ wide steel plates) at Livermore in 1997. A steel plane thickness between $2 \mathrm{~cm}$ and $4 \mathrm{~cm}$ will be selected based on energy resolution requirements and cost considerations.

Current detector optimization work is focused on the choice of active detector technology: the collaboration is developing Iarocci tube, RPC, and scintillator options in parallel. The large detector area - between 32,000 and $64,000 \mathrm{~m}^{2}$ depending on steel plane thickness - makes this the single most expensive detector component (about $\$ 15 \mathrm{M}$ for the reference detector). In addition to satisfying requirements for event topology reconstruction and energy resolution, the chosen technology must meet safety regulations for the underground laboratory (e.g., nonflammable gas), must be packaged in $2 \mathrm{~m} \times 8 \mathrm{~m}$ modules for moving down the Soudan mine shaft, and must utilize minimum floor space and assembly time during installation.

\section{3. 'Tarocci' chambers}

Iarocci tube detectors are being adapted for use in MINOS by groups at Argonne, Dubna, IHEPBeijing, Indiana, PNPI and Tufts. Previous experiments have demonstrated the successful construction and long term operation of very large arrays of Iarocci tube detectors, for both tracking and calorimetry applications. The construction cost and performance (in limited streamer mode) of these detectors are well understood [5].

The traditional Iarocci tube design has been modified for MINOS by replacing the plastic 'comb' extrusion with aluminum in order to achieve more uniform gain over the very large chamber area by tightly controlling dimensional variations. Gain uniformity is crucial because the energies of electromagnetic and hadron showers are obtained by using pulse height (charge) as a measure of the number of particles in the shower. MINOS will keep the traditional $0.9 \mathrm{~mm}$ cell size but will increase the chamber width from 8 cells to 16 cells to reduce construction and assembly costs. Figure 2 shows the current design of the chambers for MINOS.

The MINOS chambers will use $50 \mu \mathrm{m}$ anode wire and will be operated in saturated proportional mode instead of limited streamer mode. 


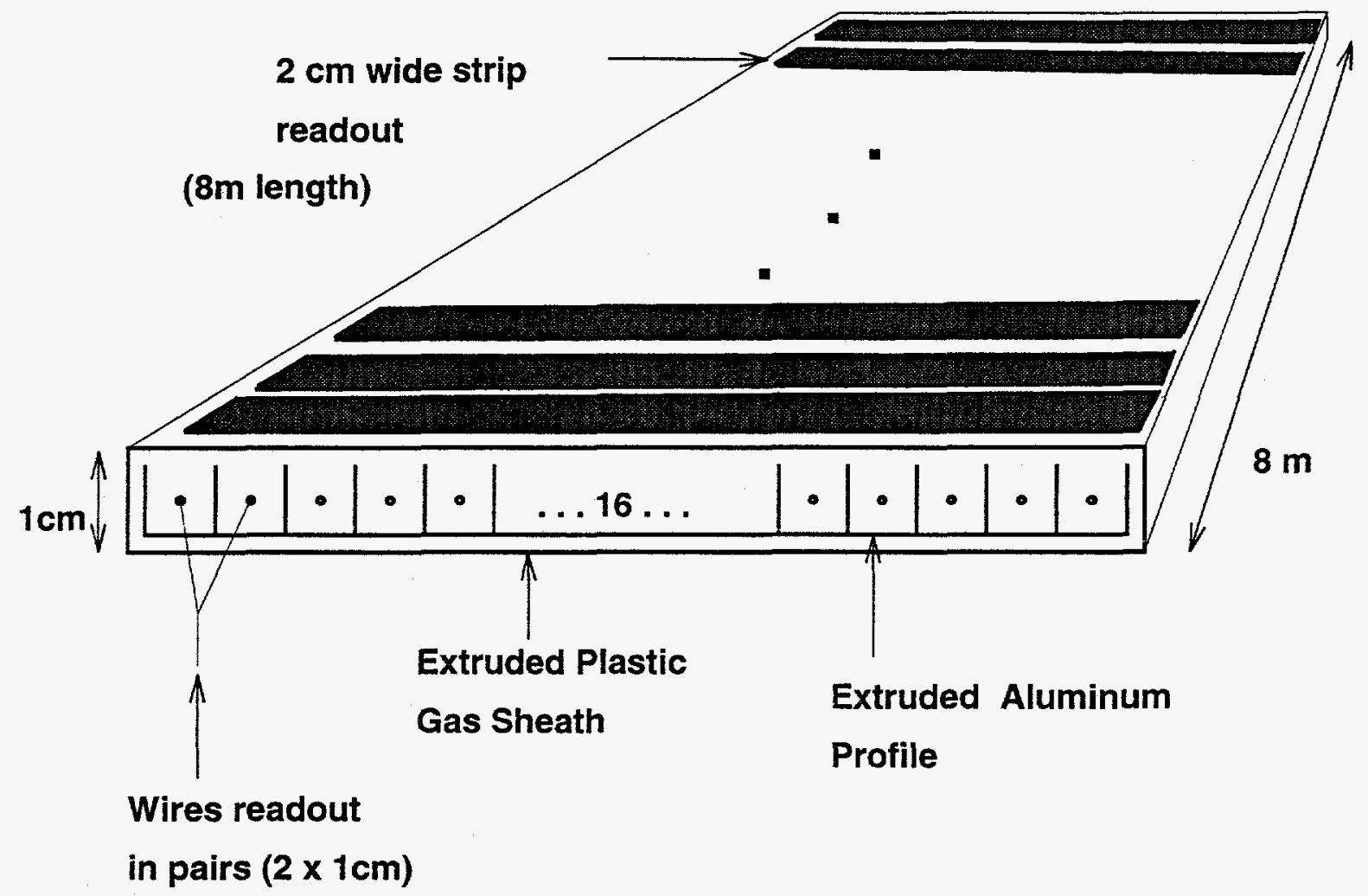

\section{'larocci' Proportional Tubes}

Figure 2. Sketch of the modified Iarocci chamber construction which has been developed for MINOS.

This allows the use of a nonflammable gas mixture: $9.5 \%$ isobutane, $88 \% \mathrm{CO}_{2}, 2.5 \%$ argon. In a SLAC test beam study the electron-shower response of single chambers, located downstream of $6 \mathrm{~cm}$ of steel, was found to be satisfactory. Full length $(8 \mathrm{~m})$ chambers of this design will be constructed and tested at Argonne during 1997.

The energy resolution of a complete calorimeter of Iarocci tubes (constructed at Dubna) was also measured during the SLAC beam tests. The EM shower response was studied in both limited streamer and saturated proportional modes for $2 \mathrm{~cm}$ and $4 \mathrm{~cm}$ thick steel plates. The shower energy was determined from the numbers of shower particles in each plane, as measured by the summed anode-wire pulse height. The expected energy resolutions were obtained and there was little difference between the two modes of operation. These results are shown in Fig. 3. The curves in the figure correspond to energy resolution fits of the form $\sigma(E)=A+B / \sqrt{E}$. For $2 \mathrm{~cm}$ steel plates, $A=0$ and $B=35 \%$ for both proportional and streamer modes. For $4 \mathrm{~cm}$ steel plates the measured resolutions were worse by $\sqrt{2}$, as expected.

The data shown in Fig. 3 indicate that the resolutions at the lowest energy are somewhat worse in proportional mode than in streamer mode. Simulations suggest that this may be due to the effect of Landau fluctuations on the ability to 


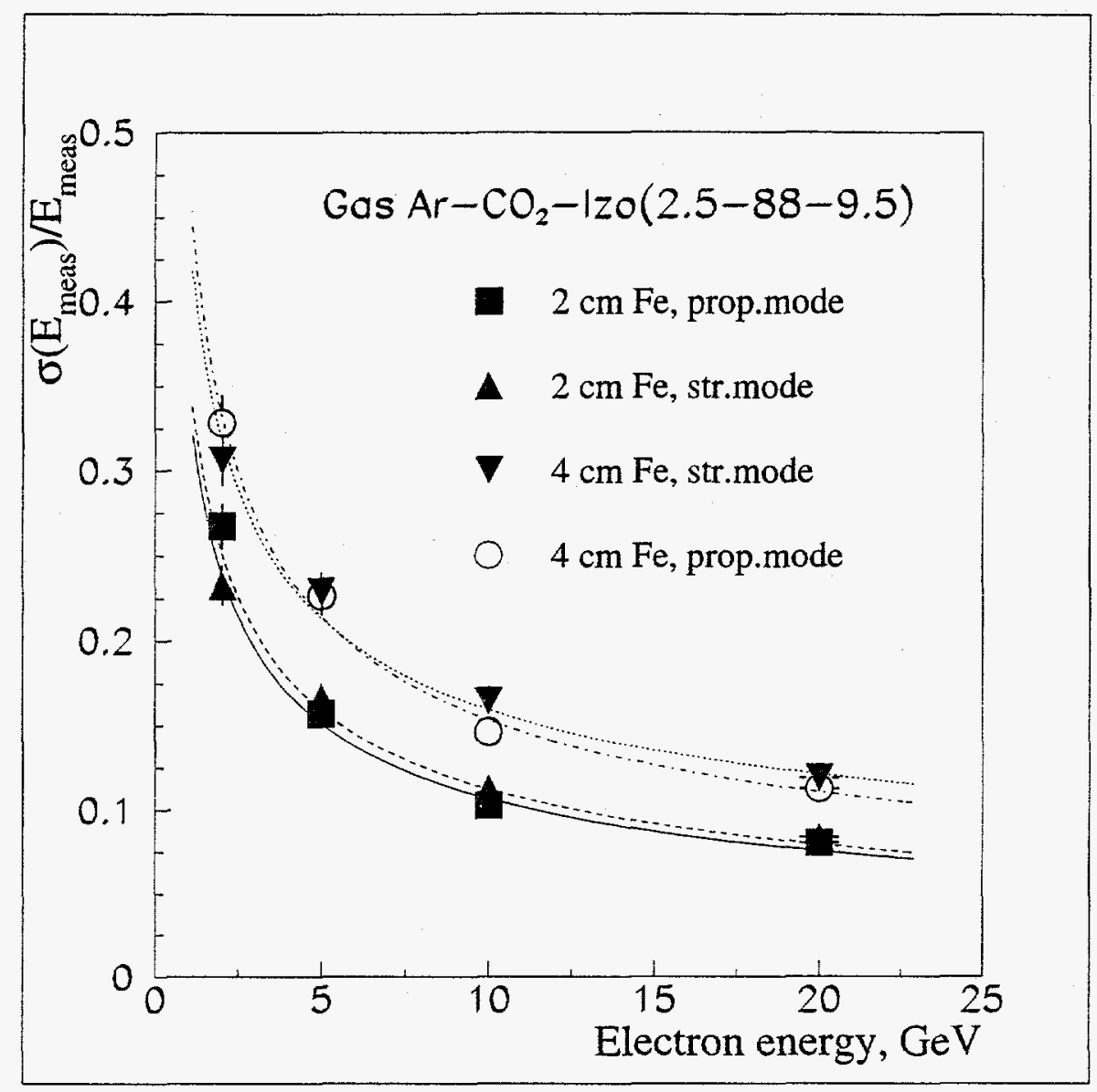

Figure 3. Energy resolutions obtained with a prototype Iarocci tube calorimeter in a SLAC test beam.

count shower particles using pulse height, which will be most pronounced in low energy showers with fewer particles per chamber plane. The very high gas gain in limited streamer mode causes a small region ( $\sim 2 \mathrm{~mm}$ radius) surrounding each initial streamer to be deadened by space charge effects. Limited streamer mode is therefore less sensitive than proportional mode to the ionization caused by the low energy delta ray electrons from Landau fluctuations.

The performance of a large prototype calorimeter, with 'Iarocci' detectors of the MINOS design, will be studied in a Fermilab test beam in 1997 with both electrons and hadrons.

\section{Resistive plate counters}

MINOS groups at Caltech, Livermore and Rutherford are developing resistive plate counters (RPC's) for MINOS using both glass [6] and ABS plastic [7] plates. Since these chambers are simply constructed and do not have anode wires, they are expected to be less expensive to build than Iarocci tubes. The small gas gap $(2 \mathrm{~mm})$ and large spark currents could make them even less sensitive to Landau fluctuations than limited streamer tubes. On the other hand, there has been much less experience with the construction and operation of very large arrays of RPC's, and virtually no ex- 


\section{MINOS GLASS RESISTIVE PLATE CHAMBERS}

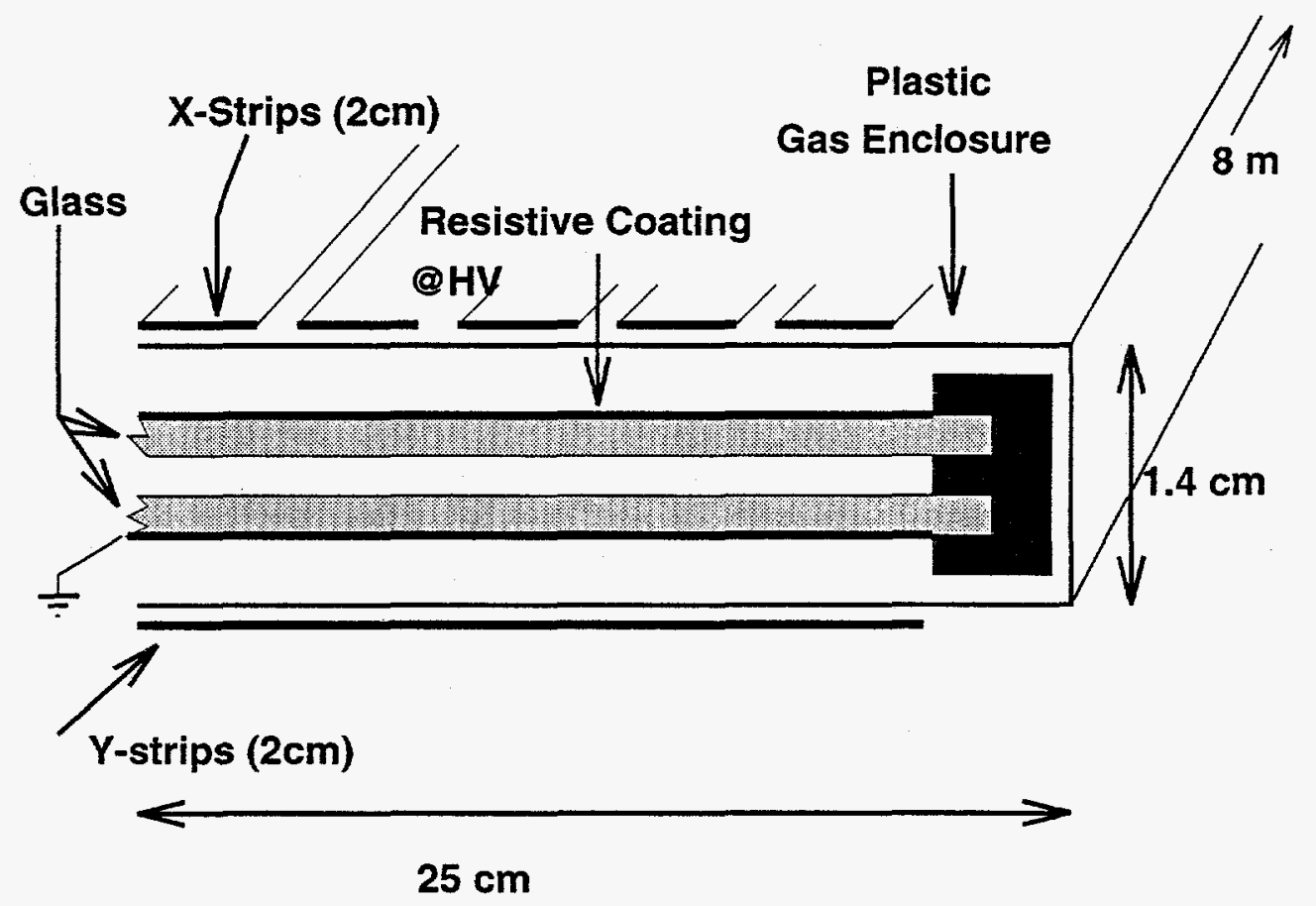

Gas: $40 \%$ Isobutane, $59 \%$ Argon, $1 \%$ SF6

Figure 4. Sketch of a prototype glass RPC chamber developed for MINOS.

perience with their use for calorimetry.

Figure 4 shows the construction of a prototype glass RPC built at Caltech. Chambers for MINOS would be $50 \mathrm{~cm}$ wide by $2 \mathrm{~m}$ long. The chamber plates are constructed of $3 \mathrm{~mm}$ thick float glass with a bulk resistivity of $\sim 10^{12} \Omega \mathrm{cm}$. The outside surfaces of the plates are coated with graphite paint to provide a resistivity of $100-1000 \mathrm{k} \Omega / \square$. The $2 \mathrm{~mm}$ separation between the glass plates is maintained by continuous edge spacers made of Delrin. Each $8 \mathrm{~m}$ diameter plane of RPC's is read out by two orthogonal arrays of $2 \mathrm{~cm}$ wide strips external to the PVC gas enclosures.

ABS plastic RPC's have been under develop- ment at Livermore since 1992 [7]. These chambers are particularly attractive for MINOS because mass production could in principle be accomplished by efficient, precisely controlled industrial processes using inexpensive commercial materials. The chamber plates are made of $0.5 \mathrm{~mm}$ thick, $1 \mathrm{~m}$ wide sheets of ABS M310 (from MiTech Corporation) supported by $0.476 \mathrm{~cm}$ thick polyurethane foam with external aluminum foil backing. The $2 \mathrm{~mm}$ chamber gap is maintained by continuous Lucite edge spacers and by an array of $1 \mathrm{~cm}$ diameter Lucite 'buttons' spaced every $10 \mathrm{~cm}$ over the active area of the plates. The resistivity of ABS M310 depends on temperature, humidity and applied voltage, but would 
be about $10^{13} \Omega \mathrm{cm}$ under RPC operating conditions. Test chambers have been constructed with a resistive graphite coating on the high-voltage ABS plate and an array of copper readout strips bonded to the other plate. Several ABS chambers have been kept in continuous, monitored operation for more than a year in a special test stand built at Livermore for longevity studies.

The usual RPC gas mixture includes both Freon 13B1 (which is no longer commercially available because of its ozone depleting effects) and isobutane (which is undesirable in the underground laboratory because of its flammability). The Livermore group has determined that $\mathrm{SF}_{6}$ can serve as an effective substitute for Freon $13 \mathrm{~B} 1$, and prototype MINOS RPC's have demonstrated good performance in SLAC electron test beam studies with a mixture of $40 \%$ isobutane, $1 \% \mathrm{SF}_{6}$ and $59 \%$ argon. Recent laboratory tests of a nonflammable mixture $(65 \%$ argon, $31 \%$ $\mathrm{R} 134 \mathrm{~A}, 4 \%$ isobutane) have also been very encouraging. (R134A is a tetraflouroethane-based ozone-friendly refrigerant.)

The afterpulsing which is commonly present in RPC signals must be eliminated or gated out if the chambers are used for calorimetry. For the SLAC test beam studies of electromagnetic shower response this was accomplished by a 30 nsec ADC gate derived from the beam trigger. For MINOS, each electronics channel would be self gated. The energy resolution obtained at SLAC from a single chamber positioned downstream of a $6 \mathrm{~cm}$ steel absorber was somewhat better than that obtained from Iarocci chambers which were being tested at the same time.

A few weeks after the end of this conference the MINOS collaboration reluctantly decided to stop work on the development of both glass and ABS RPC's, despite considerable progress and encouraging results from the SLAC test beam studies. This decision was mandated by a serious shortfall in $R \& D$ funds, and the need to focus very limited resources on fewer detector options.

\section{Scintillator}

MINOS groups at Fermilab, Minnesota, Indiana, Texas A\&M, Texas Austin, Columbia and
Protvino are developing both plastic and liquid scintillator technologies for MINOS. Compared to the gas detectors described above, scintillator is likely to provide superior energy resolution for both electromagnetic and hadronic showers. However, coarser spatial resolution could degrade the ability to reconstruct detailed event topologies and to recognize NC-like events containing electromagnetic showers. The cost of instrumenting MINOS with scintillator is perhaps the most serious concern because conventional plastic scintillator and photodetectors can be quite expensive. Since the MINOS reference detector requires 480,000 detector channels, a target of $\$ 15 /$ channel has been established for the scintillator photodetectors, which would then have a total cost of $\$ 7.2 \mathrm{M}$.

Most MINOS photodetector development to date has focused on multi-anode photomultiplier tubes. A microchannel plate device manufactured by Litton could cost as little at $\$ 13 /$ channel in a 150 channel version. Litton is currently modifying their existing design for MINOS. Other manufacturers (DEP, MELZ, Philips) also produce devices which come close to meeting MINOS requirements but, like the Litton tube, all need substantial engineering funds to make prototypes of suitable tubes.

Figure 5 shows a sketch of the liquid scintillator detector which is being developed for MINOS. Liquid-filled polypropylene tubes are read out by wavelength shifting fibers. These are spliced to clear fibers which bring the light to multi-anode photomultipliers. The $2 \mathrm{~cm}$ by $2 \mathrm{~cm}$ tubes would be up to $8 \mathrm{~m}$ long and arranged in alternating planes with orthogonal read out directions. Cost estimates based on a conceptual engineering design show that the cost of such an active detector system for MINOS, read out with multi-anode photomultipliers, may be competitive with that of a gas detector system.

Although laboratory tests of prototype liquid scintillator detectors have been very promising, substantial improvements are still possible. The reflectivity of polypropylene tubes can be improved by the addition of $\mathrm{TiO}_{2}$, and new liquid scintillator materials promise higher light yields. A new ND332 PXE-based scintillator developed 


\section{MINOS LIQUID SCINTILLATOR TUBES}

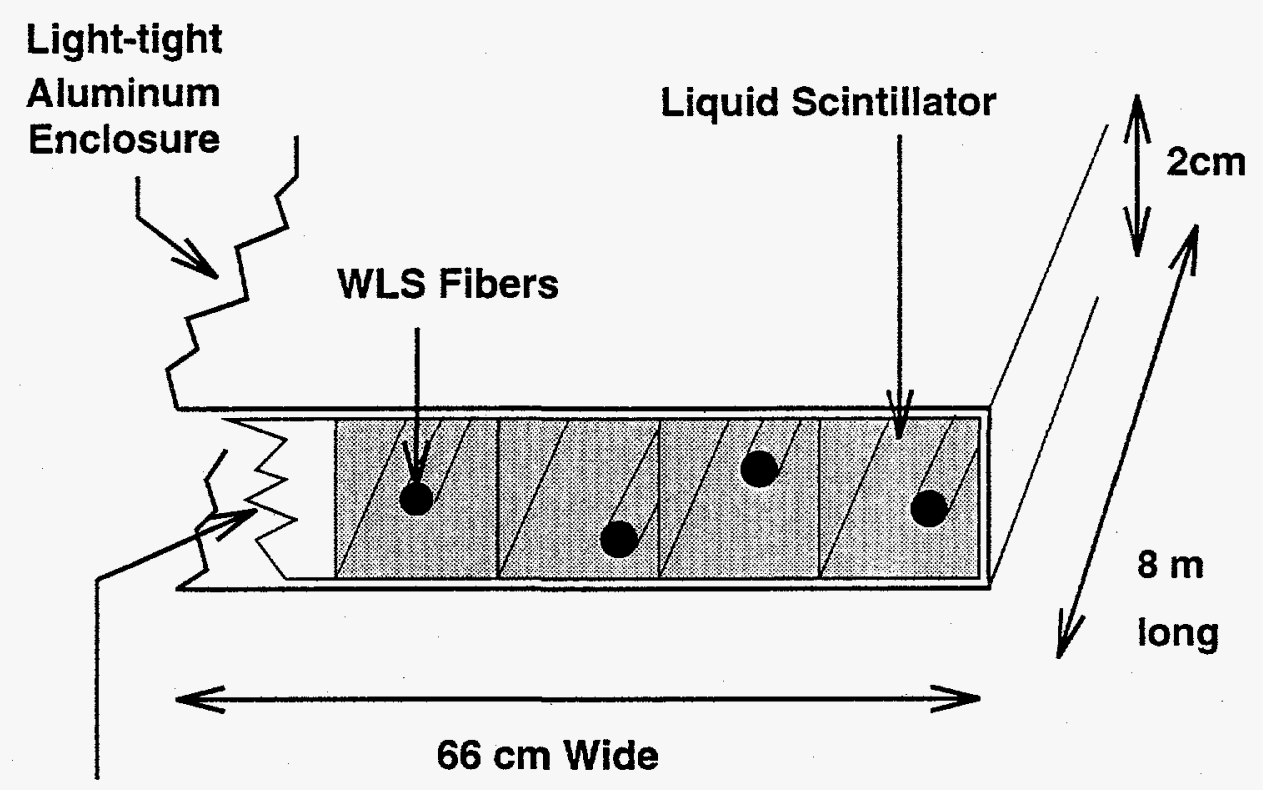

Extruded Plastic Cells

Figure 5. Sketch of a liquid scintillator detector developed for MINOS. The light collection efficiency is quite insensitive to the position of the wavelength shifting fiber within a tube.

for medical use may provide as much as 1.5 times more light than the BC517H (mineral oil plus pseudocumene) which has been used in prototype tests. After testing wavelength shifting fibers of different designs and from several manufacturers, a preliminary selection of $1 \mathrm{~mm}$ diameter multiclad BCF91A fiber from Bicron has been made. Light yield should be further increased by aluminizing the far end of the fiber.

Plastic scintillator is more convenient than liquid, but would be substantially more expensive per unit volume. Higher light yields in plastic may compensate for this by allowing thinner strips. Currently a design involving $2 \mathrm{~cm}$ by
$1 \mathrm{~cm}$ polystyrene scintillator strips is being developed along the lines of recently adopted for the preshower detector of the D0 colliding beam facility upgrade [8]. The wavelength shifting fiber readout would be very similar to that developed for the liquid scintillator technology, with the advantage that there is no concern about liquid-fiber interaction which could degrade performance over the lifetime of the experiment.

One potential advantage of scintillator technology over gas chambers with two-dimensional readout is that the two planes of scintillator detectors required to obtain two orthogonal views do not necessarily need to be located in the same 
gap between steel planes. Each $4 \mathrm{~cm}$ thick steel plane of the reference detector could be replaced by two $2 \mathrm{~cm}$ thick planes with a single plane of scintillator in each gap. This increased longitudinal segmentation would improve energy resolution without increasing the cost of the detector elements substantially. Although 1200 planes of $2 \mathrm{~cm}$ thick steel absorbers will cost more than the $6004-\mathrm{cm}$ thick planes of the reference detector, initial engineering studies show that the additional cost may not be prohibitive.

\section{Outlook}

Detailed parametrizations of the response of each detector type are being developed on the basis of prototype chamber studies with cosmic rays and in test beams, and are being incorporated into the Monte Carlo simulation of the experiment. These will be used to evaluate the sensitivity of the various oscillation tests with the different detector types under consideration. The same simulations are also essential for the optimization of the transverse segmentation (e.g., strip width) and the steel plane thickness.

The MINOS collaboration plans to choose a single active detector technology during the summer of 1997 , and to begin the setup of mass production facilities a few months later. The detector choice will be based on the projected sensitivity to oscillations of detectors instrumented with alternative technologies and built for the same total cost. Other detector issues include the performance of full scale prototype devices, the expected uniformity of response of large areas of detector, the calibration systems needed to maintain good energy resolution, and possible aging effects. Finally, conceptual engineering designs of each detector type are being developed, and will be used as a basis for cost estimates for both production and installation.

I am grateful to Yu. Gornushkin and J. Thron for their help in the preparation of this document. MINOS detector development work is supported by the U.S. Department of Energy, the U.S. National Science Foundation and the U.K. Particle Physics and Astronomy Research Council. Substantial support has also been provided by the individual universities, laboratories and research institutions of the MINOS collaboration.

\section{REFERENCES}

1. The MINOS Collaboration, "P-875: A longbaseline neutrino oscillation experiment at Fermilab," NuMI-L-63, Feb. 1995.

2. The following institutions are currently members of the MINOS Collaboration:

Argonne, Caltech, Columbia, Fermilab, IHEP-Beijing, IHEP-Protvino, Indiana, ITEP-Moscow, JINR-Dubna, Lebedev, Livermore, Minnesota, Oak Ridge, Oxford, PNPISt.Petersburg, Rutherford, Stanford, Sussex, Texas A\&M, Texas Austin, Tufts, Western Washington.

3. D.S. Ayres, "The MINOS long-baseline experiment at Fermilab," to be published in the Proceedings of the 4th KEK Topical Conference on 'Flavor Physics,' KEK, Japan, 29-31 October, 1996 (Nuclear Physics B, Proceedings Supplement).

4. L.R. Turner, "Field computation for a neutrino detector magnet: the effect of small gaps in large bodies," submitted to the Proceedings of the ICEF' 96 Conference, Wuhan, China, Oct. 1996.

5. See for example, M. de Palma, Nucl. Instr. and Meth. A277 (1989) 68-76, and G.D. Alekseev et al., Nucl. Instr. and Meth. A292 (1990) 551-562.

6. D.G. Michael and D.R. Relyea, Nucl. Instr and Meth. A373 (1996) 30-34.

7. E. Ables et al., "ABS plastic RPCs," submitted to the proceedings of the 3rd Int. Workshop on RPC's, Pavia, Italy, Oct. 1995.

8. M. Adams et al.,Nucl. Instr. and Meth. A366 (1995) 263-277. 